\title{
La calidad del empleo en la población afrodescendiente colombiana: una aproximación desde la ubicación geográfica de las comunas*
}

\author{
Recibido: 16 de abril de 2014 - Aceptado: 19 de enero de 2015
}

Doi: dx.doi.org/10.12804/rev.econ.rosario.17.02.2014.05

\author{
Lisset Pérez Marulanda ${ }^{\dagger}$ \\ Centro Internacional de Agricultura Tropical - CIAT
}

Jhon James Mora Rodríguez $\ddagger$

Universidad Icesi

\section{Resumen}

Este artículo construye un indicador compuesto sobre la calidad del empleo, al utilizar el método de componentes principales para la población afrocolombiana radicada en Cali, a partir de una encuesta representativa por comunas realizada por el Ministerio de Trabajo de Colombia. Cali, al ser la primera ciudad colombiana con mayor proporción de población afrodescendiente y la segunda de América Latina, después de Salvador Bahía, Brasil, se constituye en un referente de la situación laboral de la población afrodescendiente colombiana. Los resultados

* Los autores agradecen todos los comentarios del evaluador y del editor.

+ Máster en Economía e Investigadora en Centro Internacional de Agricultura Tropical - CIAT. Correo electrónico: lisset.perez@cgiar.org. Cali, Colombia.

$\ddagger$ Doctor en Economía, Universidad de Alcalá, Profesor Titular Economía de la Universidad Icesi, Director del ORMET-Valle del Cauca, Director, grupo de Investigación en Economía, Políticas Públicas y Métodos Cuantitativos y miembro del grupo de Investigación Alcamétrica de la Universidad de Alcalá (España). Correo electrónico: jjmora@icesi.edu.co. Cali, Colombia.

Para citar este artículo: Pérez, M. L., \& Mora, J. J. (2014). La calidad del empleo en la población afrodescendiente colombiana: una aproximación desde la ubicación geográfica de las comunas. Revista de Economía del Rosario, 17(2), 315-347. doi: dx.doi.org/10.12804/rev.econ. rosario.17.02.2014.05 
muestran que los afrocolombianos que viven en la zona Oriente, compuesta por las comunas $7,13,14,15$ y 21, tienen una mayor probabilidad de tener empleos de baja calidad, por lo que existen localizaciones espaciales en torno a la calidad del empleo. La educación universitaria aumenta la probabilidad de encontrar un empleo de alta calidad.

Clasificación JEL: J28, J7, C35

Palabras Clave: calidad del empleo, afrocolombianos, microeconometría, probit generalizado.

\title{
Job Quality in the Colombian Afro-Descendant Population: An Approach from the Geographic Location of the City Districts
}

\begin{abstract}
This article builds a compound indicator about job quality using the principal component method for the African-Colombian population resident in Cali, starting from a representative survey carried over in all city districts or "Comunas" by the Colombian Labor Ministry. Cali being the city bearing the largest proportion of Afro-descendant population in Colombia, and the second largest Afro-descendant population in Latin America after Salvador Bahia in Brazil constitutes a model for the working conditions of the Afro-descendant population in Colombia. The results show that African-Colombians living in the east side of the city, composed by districts or "Comunas" 7, 13, 14, 15 and 21 have a higher probability of having low-quality jobs, generating city areas linked to job quality. The college degrees increase the probability of finding high-quality jobs.
\end{abstract}

JEL Classification: J28, J7, C35

Keywords: job quality, african-colombian's, microeconometric, generalized probit.

\section{A Qualidade Do Emprego Na População Afrodescendente Colombiana: Uma Aproximação por Localização Geográfica das Comunas}

\section{Resumo}

Este artigo construí um indicador composto sobre a Qualidade do Emprego utilizando o método de componentes principais para a população Afro-Colombiana radicada em Cali, a partir de uma enquete representativa por comunas realizada pelo Ministério de Trabalho da Colômbia. Cali, sendo a primeira cidade colombiana com maior população afrodescendente e a segunda da América Latina, depois de Salvador Bahia no Brasil, constitui-se em um referente da situação laboral da população afrodescendente colombiana. Os resultados mostram que os afro-colombianos que moram na zona oriente composto pelas comunas 7,13,14,15 e 21 têm uma maior probabilidade de ter empregos de baixa qualidade, pelo qual existem localizações espaciais em torno à qualidade do emprego. A educação universitária aumenta a probabilidade de encontrar um emprego de alta qualidade.

Classificação JEL: J28, J7, C35

Palavras-chave: qualidade do emprego, afro-colombianos, microeconometria, probit generalizado. 


\section{Introducción}

La apertura de los mercados mundiales, la inequidad en las condiciones sociales y laborales, el deterioro de las condiciones contractuales, la tercerización del empleo y la tendencia de diferentes países a realizar reformas laborales para adecuar la legislación al nuevo entorno económico ha despertado interés entre los economistas por las cuestiones relacionadas con la calidad del empleo y las formas atípicas de emplearse (Farné, 2012).

El concepto de la calidad del empleo surge como una propuesta de marco conceptual internacional para el análisis de las condiciones laborales, y fue propuesto por la Organización Internacional del Trabajo (OIT), la Unión Europea y la Fundación Europea para la mejora de las condiciones de la Calidad de Vida y de Trabajo (Weller \& Roethlisberger, 2012). Si bien, los lineamientos de los conceptos de trabajo decente y calidad del empleo son similares, este último abarca solo algunas dimensiones del trabajo decente, puesto que incluye actividades productivas realizadas tanto por la población económicamente activa como por la inactiva (TMDMDW, 2008).

La calidad del empleo se define como el conjunto de factores vinculados al trabajo que influyen en el bienestar económico, social, psíquico y de salud de los trabajadores (Reinecke \& Valenzuela, 2000), siendo dichos factores la expresión de características objetivas, dictadas por la institucionalidad laboral y por normas de aceptación económica, social y política (Farné, 2003). Este concepto encierra además el conjunto de características personales y laborales asociadas al empleo que detentan y que se transforman en capacidades, las cuales el individuo valora porque le permiten alcanzar un mayor nivel de bienestar (Senbruch, 2007).

Cali constituye un referente de las condiciones laborales de la población afrodescendiente, puesto que - antecedida por Salvador Bahía en Brasil一, es la segunda ciudad latinoamericana con mayor proporción de población afrodesecendiente y la primera a nivel nacional. Es así como este artículo busca analizar los determinantes que inciden en la calidad del empleo en Cali, por raza, en particular, por medio de la estimación de un modelo probit ordenado generalizado. La construcción del índice y la estimación del modelo se realizaron con base en los datos de la encuesta de calidad de vida y de empleo realizada por el Ministerio de Trabajo de Colombia, entre noviembre de 2012 y enero de 2013 en la ciudad de Cali. Los resultados muestran que las comunas con menor calidad del empleo son las ubicadas al Oriente y en la ladera de la ciudad, y las comunas con mejor calidad del empleo son las ubicadas en el corredor Norte Sur, destacándose la comuna 22. Los resultados también muestran que ser 
afrodescendiente disminuye la probabilidad de tener empleos de calidad alta y media y aumenta la probabilidad de tener empleos de mala calidad.

Este artículo se divide en seis secciones, esta es la primera. La segunda sección constituye el desarrollo conceptual de los términos trabajo decente y calidad del empleo, la tercera sección presenta la revisión de la literatura sobre calidad del empleo. En la cuarta, el lector encontrará los aspectos metodológicos relacionados con esta investigación; en la quinta sección se encuentran los resultados de la construcción del Índice de Calidad del Empleo (ICE) y las estimaciones de los modelos econométricos y en la sexta están las conclusiones obtenidas de este estudio.

\section{La calidad del empleo y el trabajo decente}

En relación con el concepto de calidad del empleo utilizado en este artículo, vale la pena preguntarse ¿qué se entiende por empleo de calidad?, ¿cuáles son las diferencias entre los conceptos de trabajo decente y calidad del empleo?, ¿por qué se elige el enfoque de Calidad del Empleo? Para aclarar los anteriores interrogantes, es necesario exponer, en primer lugar, en qué consiste el concepto de trabajo decente. En 1999, la OIT, en el marco de la Conferencia Internacional del Trabajo, definió por primera vez el concepto de trabajo decente como "aquella ocupación productiva justamente remunerada y ejercida en condiciones de libertad, equidad, seguridad y respeto por la dignidad humana" (González \& Guillén, 2009). Dicho concepto está sustentando en cuatro principios: respeto a los derechos fundamentales del trabajador y a los estándares laborales internacionales, igualdad de oportunidades para todos en cuanto a empleo y remuneración, acceso a la protección social y a la seguridad social y diálogo social bipartito y tripartito (Somavia, 2004).

Ghai (2003) expone que el concepto de trabajo decente incluye la existencia de empleos suficientes (posibilidades de trabajar), la remuneración, la seguridad en el trabajo y las condiciones laborales salubres. Adicionalmente, también son elementos fundamentales la seguridad social y la seguridad de ingresos. Otros dos componentes considerados fundamentales en el concepto de trabajo decente son los derechos fundamentales del trabajo (libertad de sindicación y erradicación de la discriminación laboral, del trabajo forzoso y del trabajo infantil) y el diálogo social. Este autor también afirma que una característica importante del trabajo decente es que los trabajadores disfruten de un empleo remunerado, que es un elemento de la calidad del trabajo.

Por otro lado, el trabajo decente es caracterizado como un trabajo de calidad, cualidad que viene en reemplazo de su carácter productivo y bien remunerado 
(Uriarte, 2001). Levaggi (2006) argumenta que el concepto de trabajo decente incorpora la dimensión económica, la cual está reflejada en el concepto tradicional de un buen empleo o de un empleo de calidad, nuevas dimensiones de carácter normativo, de seguridad y de participación.

A su vez, la calidad del empleo se define como el conjunto de factores vinculados al trabajo que influyen en el bienestar económico, social, psíquico y de salud de los trabajadores (Reinecke \& Valenzuela, 2000), siendo dichos factores la expresión de características objetivas, dictadas por la institucionalidad laboral y por normas de aceptación económica, social y política (Farné, 2003). Este concepto encierra además el conjunto de características personales y laborales asociadas al empleo que detentan y que se transforman en capacidades, las cuales el individuo valora porque le permiten alcanzar un mayor nivel de bienestar (Senbruch, 2007).

Para Weller y Roethlisberger (2012), los determinantes de la calidad del empleo no solo se basan en la calidad del puesto de trabajo, la cual está relacionada con el proceso productivo, sino también por aspectos adicionales como la institucionalidad, que incide en las relaciones colectivas y de trabajo, y que influye en los ingresos, la jornada laboral, la situación contractual y la dinámica de participación, entre otros. Por tanto, es necesario incluir en su medición, tanto aspectos objetivos como subjetivos relacionados con el nivel de satisfacción del individuo con su trabajo.

De acuerdo con Guataqui, Camacho y Dussán (2012), el trabajo decente y calidad del empleo son los dos grandes enfoques en torno a la medición de la calidad del trabajo, sin embargo, ¿cuáles son las principales diferencias entre estos dos? La diferencia fundamental entre ambos consiste en que el segundo se concentra en la relación laboral de dependencia (el empleo). De esta manera, mientras que el trabajo decente se enfoca en las implicaciones sobre el bienestar de los trabajadores, la calidad del empleo se concentra en las características propias de los empleos: salarios, horas de trabajo, prestaciones sociales, tipo de contrato, etc. En este sentido, la definición de calidad del empleo difiere conceptualmente del trabajo decente, dado que trabajo y empleo son económicamente diferentes, ya que el concepto de trabajo reúne el conjunto de actividades humanas, remuneradas o no, que derivan en bienes o servicios en una economía que satisfacen necesidades o que proporcionan sustento. Por otro lado, el segundo término se refiere al trabajo desarrollado a cambio de un pago o remuneración. Por tanto, el trabajo decente es un concepto más amplio que abarca a todas las personas que participan en el mercado laboral, mientras que la calidad del empleo solo hace referencia a las características de los asalariados.

Pese a que el trabajo decente constituye una categoría de análisis más amplia, el objetivo de este artículo es fijar cuáles son los determinantes de la calidad de 
los puestos de trabajo para los asalariados en la ciudad de Cali, razón por la cual se elige el enfoque de calidad del empleo, pero se incorporan elementos que son comunes al trabajo decente como la seguridad social, la estabilidad laboral, remuneraciones, el equilibrio entre el trabajo y la vida familiar y la libertad de sindicalización. ${ }^{1}$

\section{La literatura sobre la calidad del empleo y la población afrocolombiana}

La literatura sobre la medición de la calidad del empleo y sus determinantes es relevante en este artículo, en cuanto permite conocer no solo las variables comúnmente usadas en la medición de la calidad del empleo y los factores que la determinan, sino también las principales metodologías de medición y estimación. De esta manera, la revisión de la literatura sobre este aspecto aporta tanto en la selección de indicadores para medir la calidad del empleo como para la elección de las variables que explican la probabilidad de consecución de empleos de calidad, así como también permite contrastar los resultados obtenidos con otros estudios empíricos.

En 2007, durante el cuarto seminario sobre medidas de calidad del trabajo, la OIT, la Unión Europea y la Fundación Europea para la Mejora de las Condiciones de Vida y de Trabajo compararon las dimensiones de sus marcos conceptuales y desarrollaron un nuevo marco con un enfoque internacional para la medición de la calidad del empleo. Cabe aclarar que la intención no fue crear una plantilla rígida para todos los casos, sino proponer una selección de indicadores que se podrían adaptar a cada país para reflejar las circunstancias nacionales (TMDMDW, 2008).

En el contexto regional, Posso (2010) aborda el fenómeno de la calidad del empleo desde dos perspectivas. La primera, la teoría de los mercados de trabajo segmentados y, la segunda, las mediciones empíricas propuestas por la OIT. Para ello, el autor construye un índice de calidad del empleo y estima un modelo de variable dependiente binaria que permite identificar los factores asociados con un empleo de calidad. Los resultados sugieren evidencias a favor de la teoría de la segmentación en los mercados de trabajo y en particular muestran la alta heterogeneidad que tiene la educación universitaria completa, el tipo de contratación y la localización por medio de la distribución condicionada de ingresos. Además, entre los factores asociados con un empleo de calidad se destacan la

1 Ghai (2003) usa como indicador del diálogo social el porcentaje de trabajadores asalariados que están afiliados a un sindicato. 
educación y el tamaño de la firma. Las ciudades con mejor calidad del empleo son Bogotá, Medellín y Cali y las ciudades con peor calidad del empleo son Cúcuta, Montería e Ibagué.

Mora y Ulloa (2011) estudian la calidad del empleo en las principales ciudades colombianas en 2009 y corrigen la endogeneidad de la educación mediante el método de Murphy Topel. Los autores exploran también los determinantes principales de la calidad del empleo como lo son la jefatura del hogar, el estado civil y vivir en una ciudad determinada. Como medida de la calidad del empleo, los autores construyen un índice compuesto que incluye los ingresos, el tipo de contrato, la jornada laboral, la salud, las pensiones y el subempleo. Las conclusiones obtenidas de este estudio sugieren que existen diferencias en la calidad del empleo entre hombres y mujeres y entre sectores económicos, además de la diferencia en la calidad entre ciudades. Por otra parte, existe una interesante relación entre la educación y la calidad del empleo, una mayor educación incrementa la calidad del empleo solo en el caso de los trabajadores asalariados.

Pineda (2011) estima un índice compuesto para la calidad del empleo a partir de la técnica de componentes principales y desarrolla un marco teórico en el cual considera el carácter multidimensional de la calidad del empleo y su relación con la calidad de vida. Los datos provienen de la Gran Encuesta Integrada de Hogares para las trece áreas metropolitanas del país en el segundo semestre de 2008. Para este autor, la medición de la calidad del empleo se compone de seis dimensiones. La primera, la intensidad de la jornada laboral y las condiciones de trabajo; la segunda, el ingreso; la tercera, la protección social; la cuarta, la estabilidad laboral; la quinta, es una dimensión subjetiva del trabajo, básicamente consiste en cómo se siente el individuo con su trabajo actual y finalmente la sexta, el subempleo. En relación con la construcción del índice de calidad del empleo, los resultados señalan que existe una profunda segmentación de los mercados laborales expresada en las brechas de calidad, esto constituye evidencia para replantear las políticas públicas laborales frente a las disparidades, en términos de calidad, entre grupos de trabajadores y ciudades.

Farné (2003) desarrolla un estudio sobre la calidad del empleo en Colombia en el cual se reportan los resultados de una investigación conjunta con el Observatorio del Mercado de Trabajo y la Seguridad Social de la Universidad Externado, la OIT y el DANE. Los resultados de este estudio arrojan un índice sintético de la calidad del empleo para los trabajadores asalariados e independientes, discriminados por sexo, también se presenta un índice de calidad del empleo por rama de actividad económica de los cuales se concluye que existe precariedad en las condiciones de trabajo nacionales. Por otro lado, los sectores que garantizan una buena calidad del empleo son la administración pública, el sector energético y financiero, los seguros y los servicios sociales. En relación 
con el género, no se observan diferencias significativas en la calidad del empleo de uno y otro, ambos son en promedio malos, en especial si se trata de trabajadores independientes.

En 2012, este mismo autor analiza, desde el punto de vista espacial, la calidad del empleo para Bolivia, Chile, Ecuador y Perú. En este documento, Farné realiza una revisión del estado del arte de los principales autores a nivel regional y determina cuáles son las variables sobre las que existe consenso para la medición de la calidad del empleo, estas variables son sexo, categoría ocupacional, el ingreso, la tenencia de contrato de trabajo, la afiliación al sistema de seguridad social, la jornada laboral, la antigüedad en el trabajo, la capacitación, el lugar de trabajo y el subempleo, entre otras (ver tabla 1).

Tabla 1. Variables utilizadas para el cálculo de la calidad del empleo

\begin{tabular}{|c|c|c|c|c|c|}
\hline $\begin{array}{c}\text { Variables de Calidad } \\
\text { del Empleo }\end{array}$ & Gamero & $\begin{array}{c}\text { Farné, Vergara y } \\
\text { Baquero }\end{array}$ & Senhbruch & Marull & $\begin{array}{c}\text { Weller y } \\
\text { Roethisberger }\end{array}$ \\
\hline Sexo & $x$ & $x$ & $x$ & $x$ & $x$ \\
\hline $\begin{array}{l}\text { Categoría ocupa- } \\
\text { cional }\end{array}$ & $x$ & $\mathrm{x}$ & $x$ & $x$ & $x$ \\
\hline Ingreso & $x$ & $x$ & $x$ & $\mathrm{x}$ & $x$ \\
\hline $\begin{array}{l}\text { Existencia de con- } \\
\text { trato de trabajo } \\
\text { (modalidad de } \\
\text { contratación) }\end{array}$ & $x$ & $x$ & $x$ & $x$ & $x$ \\
\hline $\begin{array}{l}\text { Seguridad social } \\
\text { (afiliación a salud } \\
\text { o pensiones) }\end{array}$ & $x$ & $\mathrm{x}$ & $x$ & $x$ & $x$ \\
\hline Jornada laboral & $x$ & $x$ & & $x$ & $x$ \\
\hline $\begin{array}{l}\text { Antigüedad en } \\
\text { el trabajo }\end{array}$ & & $\mathrm{x}$ & $x$ & & \\
\hline Subempleo & & $x$ & & $\mathrm{x}$ & \\
\hline Lugar de trabajo & & $x$ & & $\mathrm{x}$ & \\
\hline Capacitación & & & $\mathrm{x}$ & & $\mathrm{x}$ \\
\hline Otras & & $\begin{array}{l}\text { Deseo de cambiar } \\
\text { de empleo, meno- } \\
\text { res trabajadores, } \\
\text { mujeres en cargos } \\
\text { directivos }\end{array}$ & $\begin{array}{l}\text { Sindicaliza- } \\
\text { ción }\end{array}$ & $\begin{array}{l}\text { Número de } \\
\text { ocupacio- } \\
\text { nes }\end{array}$ & $\begin{array}{l}\text { Beneficios ex- } \\
\text { trasalariales, } \\
\text { vacaciones } \\
\text { pagas, sindi- } \\
\text { calismo }\end{array}$ \\
\hline
\end{tabular}

Fuente: Farné (2012). “La calidad del empleo en América Latina a principios del siglo XXI; una mirada especial a los casos de Bolivia, Chile, Colombia, Ecuador y Perú". La Calidad del Empleo en América Latina a principios del siglo XXI. Bogotá: Universidad Externado de Colombia. 
En relación con los determinantes de la calidad del empleo, los trabajos de Tenjo y Herrera (2009) y Bustamante y Arroyo (2008) incluyen en su análisis el papel de la condición étnica como determinante de la calidad del empleo. En general, encuentran que la población afrodescendiente tiene una menor probabilidad de adquirir empleos de alta calidad. En primer lugar, Tenjo y Herrera (2009) investigan la discriminación en el mercado laboral por medio de las brechas salariales entre trabajadores de sexo masculino y femenino y entre afrodescendientes y no afrodescendientes. Para ello, utilizan la Encuesta de Calidad de Vida del DANE del año 2003 y desarrollan dos ejercicios empíricos, el primero es la descomposición del salario mensual por ocupación y nivel de calificación, el cual busca conocer qué tanto de las diferencias salariales se debe a diferencias en la estructura ocupacional, y el segundo corresponde a la descomposición de Oaxaca, basada en la estimación de ecuaciones de Mincer. Encuentran que más de la mitad de las diferencias salariales promedio entre afrodescendientes y no afrodescendientes son explicadas por el hecho de que existen diferencias salariales para individuos con la misma ocupación y nivel de calificación, en general, los afrodescendientes ganan menos que el resto de los trabajadores. Por tanto, no es posible descartar la hipótesis de discriminación laboral por raza. Los resultados son similares para el género.

Adicionalmente, se ha estudiado la calidad del empleo según la raza y el género. Arroyo y Bustamante (2008) construyeron un índice de calidad del empleo para el área metropolitana de Santiago de Cali, Colombia, por medio de la metodología propuesta por Farné (2003), y analizaron los determinantes de la calidad del empleo para la población afrodescendiente, encontraron que, en general, los trabajadores afrodescendientes tienen empleos de calidad muy baja y que su condición étnica limita el acceso a empleos de mejor calidad. Por tanto, es posible pensar que hay presencia de discriminación laboral en contra de la población afro. Sin embargo, en comparación con el contexto nacional, determinan que se puede observar que, a nivel nacional, el ser de raza negra tiene un mayor efecto en las probabilidades de que tal trabajador tenga un empleo de mala calidad.

Viáfara y Urrea (2006) examinan los efectos de la raza y el género en el logro educativo y el estatus socio-ocupacional para las ciudades de Bogotá, Cartagena y Cali, para ello utilizan la Encuesta Nacional de Hogares (ENH) del DANE, etapa 110, de diciembre de 2000. Los autores encuentran que los afrocolombianos presentan un menor estatus socioeconómico familiar y que es probable que las desigualdades en la adquisición de capital escolar sean superiores, debido a la combinación de clase y raza. En términos raciales, el efecto es ligeramente más fuerte en Cali. También encuentran que para Cartagena existe mayor efecto de la interacción de raza y género. 
En relación con cuáles deben ser las estrategias para la generación de empleo en la ciudad de Cali, Mora y González (2011) proponen una estrategia de generación de empleo digno (decente y de calidad), que constituya una estrategia consensuada y coordinada por todos los agentes e instituciones que participan en el mercado de trabajo de la ciudad. Así, la elaboración de dicha propuesta política pública de empleo aporta un elemento añadido crucial para el funcionamiento del mercado laboral en el futuro y para que los actores y la población inviertan sus iniciativas y capacidades en la economía formal y no en la economía informal. Esta propuesta se basa en las siguientes actuaciones de política: (a) fortalecer la educación y la capacitación de los recursos humanos para que actúen como principal impulsor de la innovación tecnológica y de la economía formal, (b) mejorar la coordinación y el diálogo institucional, (c) potenciar la regeneración urbana integral de la ciudad e integrarla alrededor del Masivo Integrado de Occidente (MIO), (d) potenciar la generación de estructuras que favorezcan el empleo. El objetivo es generar las estructuras básicas necesarias para el buen funcionamiento de la sociedad y de la economía de la ciudad, que tengan repercusión en la productividad del tejido productivo, para ellos se propone la promoción de zonas prioritarias de actividad económica, el acceso de las pequeñas y medianas empresas a los microcréditos y el mejoramiento del clima de los negocios en la región. (e) Favorecer la cultura emprendedora y los sectores de la economía formal con potencial de futuro y (f) desarrollar políticas de rentas compensatorias para mejorar los niveles de subsistencia de la población más desfavorecida. El desarrollo de estos aspectos debe estar cruzado transversalmente por el consenso entre los principales actores institucionales, económicos y sociales, públicos y privados en torno a las propuestas de creación activa de empleo y por el liderazgo del Estado.

Por otro lado, en Bogotá, bajo el marco de Política Pública de Productividad, Competitividad y Desarrollo Socioeconómico, Guataqui, et al. (2012) desarrollaron un estudio sobre las condiciones de la calidad del empleo en la capital y, partiendo de su análisis, desarrollaron diversas propuestas de política pública que permitan mejorarlas. Los autores eligen el enfoque de trabajo decente, basados en el hecho de que el concepto de trabajo es más amplio que el concepto de empleo, puesto que este último solo abarca los trabajadores asalariados. A partir de la Gran Encuesta Integrada de Hogares (GEIH) para los años 2007 a 2010, los autores calculan un Índice Compuesto de Trabajo Decente (ICTD), propuesto por Bescond et al. (2003), y encuentran que, durante el periodo analizado, la calidad del empleo mejoró al igual que el conjunto de indicadores usados en su cálculo. Los autores también realizan un Análisis por Dimensiones del Trabajo Decente y evalúan el estado de cada componente, en este aspecto, los resultados no son alentadores, los ingresos reales de los trabajadores de la capital exhiben un de- 
crecimiento en el periodo de estudio, al tiempo que se observa una reducción de las oportunidades laborales asalariadas y formales frente a las no asalariadas e informales. Por otro lado, construyen un Índice Compuesto de Calidad del Empleo (ICCE) por medio de la técnica de media truncada, para el cual se observa una tendencia a la baja en el largo plazo tanto para Bogotá como para las trece áreas metropolitanas, indicando avances de política, puesto que este es un índice de carencia de oportunidades.

Finalmente, otro resultado de este estudio es el hecho de que a mayor nivel educativo menores tasas de desempleo y menor el tiempo de espera para encontrar un trabajo. En concordancia con estos resultados, este estudio promociona la importancia de la educación superior porque promueve el acceso a empleos de mejor calidad, en este sentido, el incremento de la calidad del trabajo en Bogotá exige medidas de política dirigidas al incremento del capital humano, especialmente en la población más vulnerable. Los autores proponen además que las autoridades locales propendan por la retención de la población en edad escolar, mayores condiciones de empleabilidad para los jóvenes; evaluación de la calidad y pertinencia de la educación superior y formación para el trabajo; identificación de las dinámicas migratorias de los individuos con educación superior e incrementos de la productividad empresarial.

Por otro lado, el efecto de las interacciones sociales a una escala geográfica también es relevante en los resultados en el mercado de trabajo. Brock y Durlauf (2002) desarrollan un modelo teórico de toma de decisiones en presencia de efectos vecindario. Por efecto vecindario se entiende la interdependencia entre las decisiones individuales y las características comunes a los individuos que residen en su mismo vecindario. En este sentido, cada agente forma sus expectativas de acuerdo con el porcentaje de agentes, quienes han tomado la misma decisión en su vecindario. Los autores plantean que en el trabajo empírico los incentivos privados están determinados por dos tipos de variables: un vector de características individuales y un vector de características del vecindario. En este sentido, es desarrollado el trabajo de Bayer, Ross y Topa (2005), quienes analizan las interacciones sociales en una escala geográfica determinada y sus efectos en el mercado de trabajo, al usar datos del Censo del área metropolitana de la ciudad de Boston, desarrollan un ejercicio empírico controlado por efectos fijos y características socioeconómicas de los individuos. Los autores examinan cómo la cercanía del lugar de residencia puede incrementar la probabilidad de compartir el mismo lugar de trabajo.

Bayer et al. (2005) encuentran que para dos individuos que residan en el mismo vecindario, la probabilidad de trabajar juntos es mayor en 6,9 puntos porcentuales de lo que podría ser si no tuvieran una relación geográfica cercana y ausencia de referencias laborales. Existe evidencia del impacto de la 
información sobre trabajos, proporcionada por medio de las redes sociales en diferentes grupos demográficos. Los jóvenes que tienen poca educación y son de género masculino, son más propensos a usar redes sociales informales para conseguir un trabajo. Así pues, los autores encuentran un efecto "referencias", el cual es altamente significativo y positivo, lo que induce una mayor probabilidad de que los individuos trabajen juntos. Esto indica que si una persona reside en un lugar en el cual una gran proporción de individuos tienen unas condiciones del empleo precarias, y son estos individuos quienes conforman su red social y por tanto sus referencias laborales, es probable que encuentre un trabajo en estas mismas condiciones. Es decir, para estos autores, la calidad del puesto de trabajo de una persona empleada (la calidad del emparejamiento en el mercado laboral) está determinada por la calidad de las redes sociales y referencias laborales establecidas con personas empleadas que residen en su vecindario. Bayer, et al. (2005) resaltan que las interacciones sociales tienden a ser más fuertes cuando los individuos que interactúan son similares en términos de educación, edad, presencia de hijos, participación en el mercado de trabajo y estado civil; características que pueden ser comunes a lo largo del vecindario a causa de la segregación.

En resumen, la revisión de la literatura da luces no solo de los principales conceptos, definiciones de la calidad del empleo sino también de las variables explicativas relacionadas y de los esfuerzos y enfoques para medirla. En general, existe consenso sobre algunas variables que se consideran explicativas de la calidad del empleo como lo son horas trabajadas, salarios, afiliación a la seguridad social y pensiones y variables de tipo subjetivo que reflejan la conformidad del individuo con las condiciones laborales. Además, los diferentes ejercicios empíricos sugieren que es posible que existan diferencias en la calidad del empleo entre zonas geográficas y entre grupos sociales. También, permite identificar, por medio de los resultados empíricos de los estudios considerados, el papel de la condición étnica en la calidad del empleo. Finalmente, se resalta la importancia de reconocer los efectos de las interacciones sociales a una escala geográfica determinada, la cual en este caso será a nivel de comuna, y sus efectos en el mercado laboral. Por otro lado, se consideran recomendaciones de política pública dirigidas a mejorar la calidad del empleo.

\section{Metodología}

Al igual que Pineda (2011), aquí se utilizará un índice de calidad del empleo construido a partir del método de componentes principales. La tabla 2 muestra las variables incluidas en la construcción del índice de calidad del empleo aquí 
propuesto. Las variables tipos de contrato, vacaciones pagas, prima de navidad, derecho a cesantías, salarios, afiliación a una organización sindical, aseguramiento al sistema de salud y pensiones, estabilidad laboral y compatibilidad de la jornada laboral con las relaciones familiares, tienen un efecto positivo sobre el índice calculado, mientras que el número de horas trabajadas tienen un efecto negativo sobre el valor del índice si se superan las 48 reglamentadas en la legislación laboral colombiana.

Tabla 2. Variables incluidas en la construcción del índice de calidad del empleo (ICE)

\begin{tabular}{ll}
\hline \multicolumn{1}{c}{ Variable } & \multicolumn{1}{c}{ Descripción } \\
\hline Tipo de contrato & $\begin{array}{l}\text { Personas encuestadas con un tipo de contrato a término fijo o } \\
\text { indefinido }\end{array}$ \\
Vacaciones pagas & Personas encuestadas con derecho a vacaciones pagas \\
Prima navidad & Personas encuestadas con derecho a prima de navidad \\
Derecho Cesantías & Personas encuestadas con derecho a cesantías \\
Salarios & Salario promedio mensual (COP) \\
Asegurado sistema de salud & Personas afiliadas a salud y pensiones en cada comuna \\
y pensiones & Personas encuestadas que consideran que su trabajo es esta- \\
Estabilidad laboral & ble o muy estable \\
Compatibilidad de la jornada & $\begin{array}{l}\text { Personas encuestadas en cada comuna que consideran que su } \\
\text { jornada laboral es compatible con sus relaciones familiares }\end{array}$ \\
Horas trabajadas semana & Promedio de horas trabajadas a la semana \\
Afiliación sindical & Personas afiliadas a una organización sindical \\
\hline
\end{tabular}

Fuente: elaboración propia.

El tipo de contrato, las vacaciones pagas, el derecho a una prima de navidad, la afiliación al sistema de seguridad social y el derecho a cesantías representan beneficios asociados con la estabilidad laboral del empleado. Por otro lado, los salarios son considerados en la literatura como uno de los principales factores que inciden en la calidad del empleo; las horas trabajadas a la semana se convierten en un factor negativo si estas exceden las 48 horas reglamentadas en la legislación laboral colombiana. Finalmente, se incluyen dos variables de carácter subjetivo, el número de personas encuestadas que consideran que su trabajo es estable o muy estable y el número de personas encuestadas que consideran que su jornada laboral está acorde con su vida familiar.

A los valores tipificados de las variables seleccionadas se les aplica la técnica de análisis multivariante de componentes principales. Una vez extraídos los componentes y sus tasas de contribución, se procede a evaluar cada una de 
las unidades experimentales de acuerdo con el índice. De esta forma, se tiene en cuenta una ecuación general que consiste en un promedio ponderado de las puntuaciones de cada componente principal, ponderados por la raíz cuadrada de la varianza de cada componente (Peters et al., 1970). Dado lo anterior, el índice para cada unidad experimental (comuna) se calcula como:

$$
I_{m i}=\frac{\sum_{i=1}^{r} Z_{r j} \cdot \sqrt{\lambda_{r}}}{\sum_{i=1}^{r} \sqrt{\lambda_{r}}}
$$

Donde $I_{m j}$ representa el índice para cada unidad experimental $j ; Z_{r j}$ es la puntuación del componente r-ésimo para la unidad experimental j-ésima y $\sqrt{\lambda_{r}}$ es la raíz cuadrada del autovalor para dicho componente, esto garantiza que los componentes con una mayor varianza explicada tengan una mayor ponderación en la calificación del índice. Dado lo anterior, cabe destacar que la construcción del índice es objetiva en cuanto dichos pesos no son determinados por el investigador si no son establecidos por la varianza explicada de cada componente.

A continuación, se discretizó el índice en tres rangos: calidad alta, calidad media y calidad baja siguiendo la metodología de Farné (2003) y Mora y Ulloa (2011). Según Mora y Nuñez (2010), la forma de discretización de la variable dependiente influye sobre la distribución de esta y, por ende, sobre los resultados de las estimaciones, por lo tanto, la forma de discretización elegida para el análisis obedece a razones teóricas de interés para el investigador. En este artículo, se presentan los resultados del análisis de los determinantes de la calidad del empleo, siguiendo la metodología de Farné (2003), la cual se considera estándar en la literatura colombiana relacionada con el tema de interés, se realiza la discretización del índice convirtiéndolo a un rango de 0 a 100 y estableciendo los umbrales según las calificaciones del índice de 0 a 60 puntos, calidad baja; de 60 a 80, calidad media y de 80 a 100 puntos, calidad alta.

De esta forma, el índice no es continuo sino discreto y presenta tres niveles. Teniendo en cuenta lo anterior, el modelo econométrico es:

$$
y^{*}=X^{T} \beta+\varepsilon
$$

Donde $y^{*}$ no es observada, pero se observa que 


$$
\begin{aligned}
& y=0 \text { si } y^{*} \leq 0 \\
& y=1 \text { si } 0<y^{*} \mu_{1} \\
& y=2 \text { si } \mu_{1}<y^{*}<\mu_{2} \\
& \cdots \\
& y=J \text { si } \mu_{j-1} \leq y^{*}
\end{aligned}
$$

$\mu$ es un parámetro desconocido, se asume que $\varepsilon \sim N(0,1)$. Dado lo anterior, se tienen las siguientes probabilidades:

$$
\begin{aligned}
& \operatorname{Pr}(y=0 \mid X)=\Phi\left(-x^{\prime} \beta\right), \\
& \operatorname{Pr}(y=1 \mid X)=\Phi\left(\mu_{1}-x^{\prime} \beta\right)-\Phi\left(-x^{\prime} \beta\right) \\
& \operatorname{Pr}(y=2 \mid X)=\Phi\left(\mu_{2}-x^{\prime} \beta\right)-\Phi\left(\mu_{1}-x^{\prime} \beta\right) \\
& \cdots \\
& \operatorname{Pr}(y=J \mid X)=1-\Phi\left(\mu_{j-1}-x^{\prime} \beta\right)
\end{aligned}
$$

Para que estas probabilidades sean positivas debe ser cierto que: $0<\mu_{1}<\mu_{2}<\ldots<\mu_{j-1}$

Los parámetros son obtenidos mediante la siguiente razón de verosimilitud

$$
L=\sum_{i=1}^{N} \sum_{j=1}^{m} y_{i j} \ln p_{i j}
$$

Siendo $p_{i j}$ las probabilidades definidas en (4)

Para aplicar correctamente el modelo probit ordenado, especificado en las ecuaciones (2) a (5), debe cumplirse el supuesto de regresiones paralelas sobre el modelo de elección discreta no ordenado, lo que implica que este es definido como un conjunto de modelos de elecciones binarias con diferente constante pero con la misma pendiente.

\subsection{El supuesto de regresiones paralelas y el test de Brant}

Partiendo de la formulación de un modelo ordenado de elección discreta (Long, 1997), se tiene que:

$$
\operatorname{Pr}\left(\mathrm{y} \geq J \mid x_{i}\right)=\mathrm{F}\left(\mu_{j}-\beta^{\prime} x_{i}\right) ; j=1, \ldots, J-1
$$

diferenciando respecto a $x_{i}$ se obtiene

$$
\partial \operatorname{Pr}\left[y_{i} \leq j \mid x_{i}\right] / \partial x_{i}=-f^{*}\left(\mu_{i}-\beta x_{i}\right) \beta
$$


Esto se define como un conjunto de modelos de elecciones binarias con diferente constante pero con la pendiente, $\beta$ en común. Si se fija la probabilidad $P=P^{*}$ para cualquier resultado, se tiene que por monotonicidad de la función de densidad normal, la función $f\left(\mu_{i}-\beta x_{i}\right)$ es fija para $f^{*}$. Esto es, la probabilidad de una elección particular es:

$$
\partial \operatorname{Pr}\left[y_{i} \leq j \mid x_{i}\right] / \partial x_{i}=f^{*} \beta=\partial \operatorname{Pr}\left[y_{i} \leq \mathrm{m} \mid x_{i}\right] / \partial x, m=0, \ldots, J
$$

Donde $f^{*}$ es igual para todo $J$, o lo mismo, es un múltiplo de $\beta$. Esta característica intrínseca del modelo ordenado es llamada supuesto de regresiones paralelas.

Brant (1990) se aproxima a la cuestión de las regresiones paralelas, el test, conocido como el Test de las Odds Proporcionales toma como hipótesis nula $\beta_{1}=\beta_{2} \ldots=\beta_{J-1}$ lo que implica que $\operatorname{Pr}\left(\mathrm{y} \geq j \mid x_{i}\right)=\phi\left(\beta_{0 j}-\beta^{\prime} x_{i}\right)$ donde $\beta_{0 j}$ $=\beta_{0}-\mu_{j}$ y $\phi$ es la función de densidad normal. El vector de pendientes de la ecuación $\beta_{j}$ podría ser el mismo en cada ecuación. Esta especificación implica que J-1 modelos de elección binaria pueden ser estimados al tiempo. Cada uno con una constante y el mismo vector de pendientes. La hipótesis nula es equivalente a

$$
H_{0}: \beta_{q}-\beta_{1}=0, q=2, \ldots, J-1
$$

La cual puede ser resumida como:

$$
H_{0}: R \beta^{*}=0 \text {, donde }
$$

$$
R=\left[\begin{array}{ccccc}
I & -I & 0 & \ldots & 0 \\
I & 0 & -I & \ldots & 0 \\
\ldots & \ldots & \ldots & \ldots & \ldots \\
I & 0 & 0 & \ldots & -I
\end{array}\right] \quad \beta^{*}=\left[\begin{array}{c}
\beta_{1} \\
\beta_{2} \\
\ldots \\
\beta_{k}
\end{array}\right]
$$

El estadístico de Wald asociado con esta prueba sigue una distribución chicuadrado y se define como

$$
\chi^{2}[(J-1) K]=\left(R \hat{\beta}^{*}\right)^{\prime}\left[R \times A \operatorname{Asy} \cdot \operatorname{Var}[\hat{\beta} *] R^{\prime}\right]^{-1}\left(R \hat{\beta}^{*}\right)
$$


Donde $\hat{\beta}^{*}$ se obtiene de los estimadores individuales de los probit binarios de $\beta$ (sin el término constante). Usando los resultados del test de Brant o test de las odds paraleas, la matriz de varianzas y covarianzas asintótica se define como:

$$
\begin{aligned}
& \text { Est.Cov.Asy }\left[\beta_{i}, \beta_{j}\right]=\left[\sum_{i=1}^{n} \hat{\Phi}_{i j}\left(1-\hat{\Phi}_{i j}\right) x_{i} x_{i}^{\prime}\right]^{-1}\left[\sum_{i=1}^{n} \hat{\Phi}_{i m}\left(1-\hat{\Phi}_{i j}\right) x_{i} x_{i}^{\prime}\right]\left[\sum_{i=1}^{n} \hat{\Phi}_{i m}\left(1-\hat{\Phi}_{i m}\right) x_{i} x_{i}^{\prime}\right]^{-1} \\
& Y \hat{\Phi}_{i j}=\Phi\left(\hat{\beta}_{0 j}-\hat{\beta}^{\prime} x_{i}\right) .
\end{aligned}
$$

Si se rechaza la hipótesis nula de este test, asumiendo que el supuesto de regresiones paralelas no se cumple y se estima el modelo probit, puede ocurrir: (a) mala especificación de la variable latente, $\beta^{\prime} x$ (probabilidades negativas), (b) heterocedasticidad de $\varepsilon$ y, finalmente, (c) mala especificación de la distribución de la variable latente (por ejemplo, esta puede no seguir una distribución logística o normal). Por lo tanto, si se rechaza la hipótesis nula debe estimarse un probit ordenado generalizado (Mora \& González, 2013).

\subsection{El modelo probit ordenado generalizado}

Cuando no se cumple el supuesto de regresiones paralelas en un modelo probit ordenado, la forma alternativa sugerida para la especificación del modelo es (González et. al., 2013; Grenee, 2010; Cameron \& Trivedi, 2005):

$$
y_{i}^{*}=\alpha+\beta^{\prime} x_{i}+\varepsilon
$$

Donde

$$
\begin{aligned}
& y_{i}=0 \text { si } \quad y_{i}^{*} \leq 0 \\
& y_{i}=1 \text { si } 0<y_{i}^{*} \leq \mu+\delta^{\prime} x_{i} \\
& y_{i}=2 \text { si } y_{i}^{*} \geq \mu+\delta^{\prime} x_{i}
\end{aligned}
$$

Si el primer elemento es normalizado a cero, $\mu_{0}=0$, se tienen las siguientes probabilidades

$$
\begin{aligned}
& \operatorname{Pr}(y=0 \mid x)=\Phi\left(-\alpha-\beta^{\prime} x\right)=1-\Phi\left(\alpha_{0}+\beta_{0}{ }^{\prime} x\right) \\
& \operatorname{Pr}(y=1 \mid x)=\Phi\left(\mu+\delta^{\prime} x_{i}-\alpha-\beta^{\prime} x\right)-\Phi\left(-\alpha-\beta^{\prime} x\right)=\Phi\left(\alpha_{0}-\beta_{0}{ }^{\prime} x\right)-\Phi\left(\alpha_{1}-\beta_{1}{ }^{\prime} x\right) \\
& \operatorname{Pr}(y=2 \mid x)=\Phi\left(\alpha+\beta^{\prime} x-\mu-\delta^{\prime} x\right)=\Phi\left(\alpha_{1}-\beta_{1}{ }^{\prime} x\right)
\end{aligned}
$$


Donde $\alpha_{0}=\alpha, \beta_{0}=\beta, \alpha_{1}=\alpha-\mu, \beta_{1}=(\beta-\delta)$ es denominado el modelo probit ordenado generalizado (Grenee, 2010), en el que se reconoce el no cumplimiento del supuesto de regresiones paralelas. Por lo tanto, la generalización permite obtener un vector de parámetros diferente para cada resultado. Así pues, la especificación general será $\operatorname{Pr}\left(y_{i}=j \mid x_{i}\right)=F\left(\mu_{j-1}-\beta_{j-1}{ }^{\prime} x_{i}\right)-\mathrm{F}\left(\mu_{j-1}-\beta_{j-1}{ }^{\prime} x_{i}\right)$ donde $F\left(\right.$.) corresponde a la función de densidad normal y $y_{i}$ es la variable dependiente, ordenada, de elección discreta, definida en Farné (2012). y $\mu_{j}$ es un umbral definido para todos los individuos de la muestra.

Por otra parte, $Y_{i}^{*}$ es la calidad del empleo alta, media y baja. En las variables explicativas se encuentran el sexo, la cual toma un valor de 1 y 0 si es mujer; estado civil, toma un valor de uno si está casado o vive en unión libre y 0 en caso contrario; jefe de hogar, toma un valor de 1 si es jefe de hogar y 0 en caso contrario; la pertenencia a una determinada comuna y la edad. Las variables: bachiller, técnico y universitario y posgrado son variables binarias, que corresponden al máximo título alcanzado por el individuo y toman el valor de uno si ocurre el evento y cero en caso contrario. Se incluyen además variables categóricas para el sector $\mathrm{u}$ actividad económica en la cual se desempeñe el ocupado, una variable dummy para las personas que pidieron ayuda a sus amigos y/o familiares para encontrar su trabajo y las interacciones de esta última con el lugar de residencia.

\section{Datos y resultados}

Los datos fueron tomados de la encuesta de Calidad de Vida y Empleo para Cali. Esta encuesta fue realizada por el Ministerio del Trabajo en asocio con la Alcaldía de Cali, la Cámara de Comercio de Cali, la Universidad Icesi y la Universidad del Valle a finales de 2012 y comienzos de 2013 en las veintidós comunas de la ciudad. Esta encuesta es representativa para cada una de las comunas y fue aplicada a 8600 hogares y un total de 30458 personas. La tabla 3 presenta algunas estadísticas descriptivas de las variables de la encuesta. En esta encuesta se incluyeron 10769 ocupados en las veintidós comunas y corregimientos de la ciudad, de los cuales el $58 \%$ son hombres y el $42 \%$ son mujeres, de esta población el $78 \%$ es no afro y el $21 \%$ es afrodescendiente, el $40 \%$ de las personas encuestadas tienen un tipo de contrato fijo o indefinido. Aproximadamente el 34,9\% de los encuestados tienen derecho a vacaciones pagas y a prima navideña y el $35 \%$ a cesantías. El $44 \%$ de las personas encuestadas están afiliadas al sistema de seguridad social y el $2 \%$ pertenece a una organización sindical. 


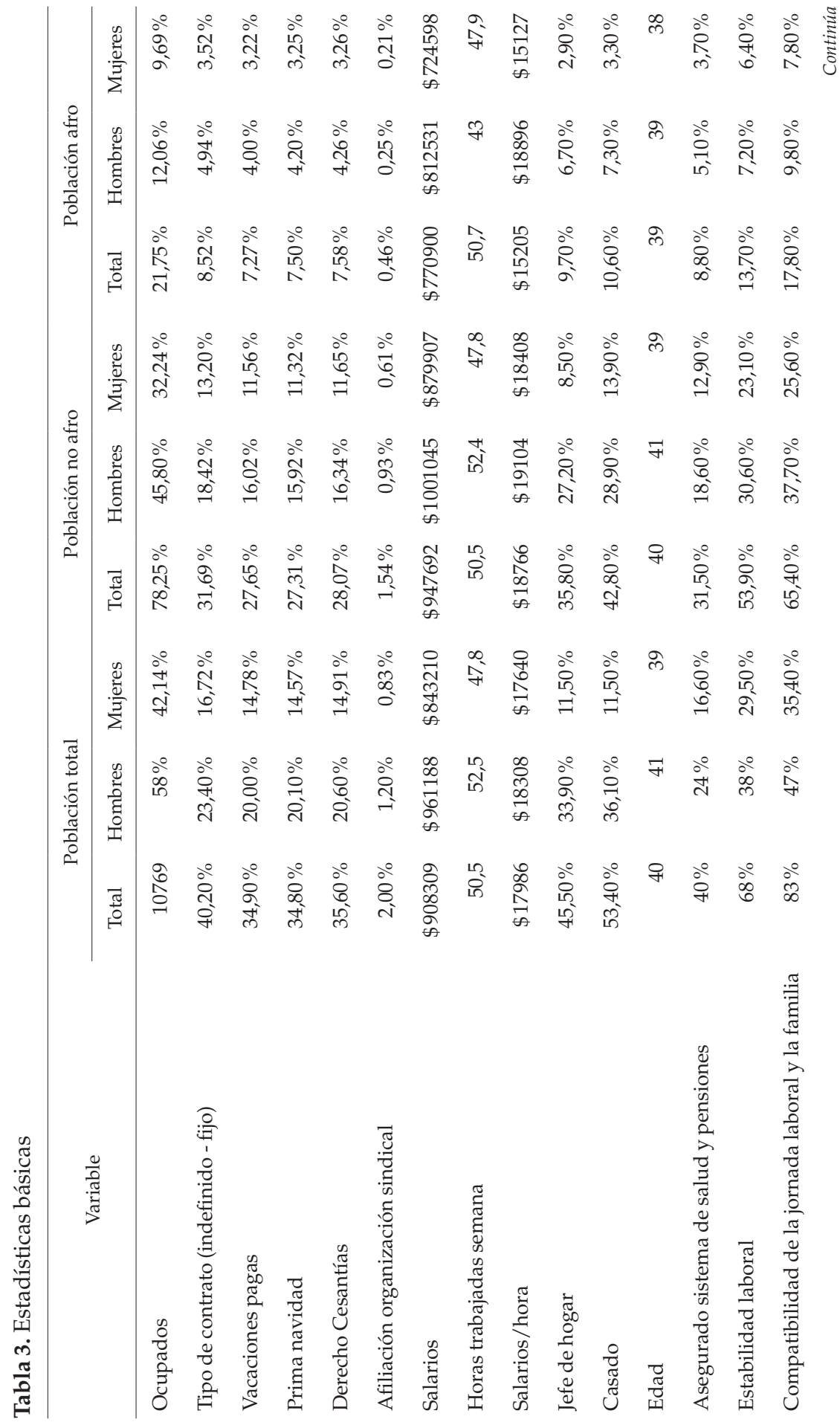




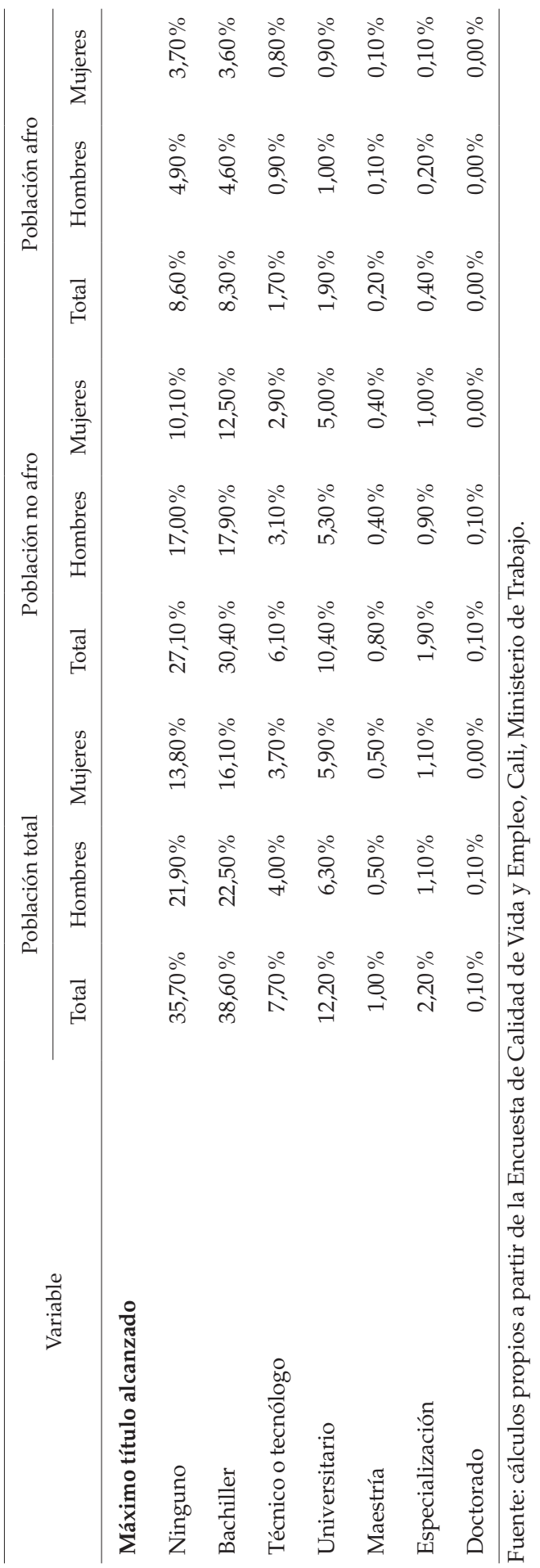


El promedio salarial en Santiago de Cali es de $\$ 908309$ pesos para el total de la población, de $\$ 961188$ para los hombres y de $\$ 843210$ para las mujeres, llama la atención que para la población no afro el salario promedio sea de \$947692, mientras que para los afrodescendientes el promedio salarial es $\$ 770900$. El porcentaje de personas que considera que su trabajo es estable y que es compatible con sus relaciones familiares es $68 \%$ y $83 \%$, respectivamente. El promedio de horas trabajadas a la semana es 50,5 y la edad promedio es de 40 años. El salario promedio por hora es en general menor para la población afrodescendiente, que gana \$15205 por hora trabajada, mientras que la población no afro gana 18766 y el total \$17986. El 45,5\% de las personas son jefes de hogar y el 53\% es casado o vive en unión libre. Respecto al nivel educativo, el 35,7\% de los encuestados no tenía ningún nivel de educación, 38,6\% título de bachiller, el 7,7\% son técnicos o tecnólogos, el 12,2\% han alcanzado un título universitario, el 1\% maestría, el 2,2\% especialización y $0,1 \%$ tiene un doctorado.

Después de construido, el índice de calidad del empleo se estandarizó entre 0 y 100. Siguiendo el establecimiento de las categorías de la calidad del empleo propuestas por Farné (2003), un índice de calidad del empleo bajo se encuentra entre 0 y 60, un índice de calidad del empleo medio entre 60 y 80 y uno alto entre 80 y 100. La tabla 4 y la figura 1 muestran los resultados del ICE construido para las veintidós comunas de Santiago de Cali. Se observa que dieciocho de las veintidós comunas de la ciudad presentan un ICE bajo, destacándose por el bajo valor del índice las comunas 20,12,13 y 16. Estas comunas se componen de barrios como Siloé, El Cortijo, Belisario Caicedo, Lleras Camargo, Belén, Brisas de Mayo, Villanueva, Asturias, Eduardo Santos, Alfonso Barberena, El Paraíso, Fenalco Kennedy, El Poblado I y II, Comuneros, Villa del Lago, Lleras Restrepo, El Vergel, El Diamante, Ricardo Balcázar, Omar Torrijos, Los Robles, Mariano Ramos, República de Israel, Unión de Vivienda Popular y Antonio Nariño. Las comunas con una calidad del empleo media son la 5, 19 y 17 conformadas por barrios como El Sena, Los Andes, Los Guayacanes, Chiminangos etapas I y II, Metropolitano del Norte, Santa Anita, La Selva, El Ingenio, Ciudad Capri, Las Quintas de Don Simón, Mayapán, Caney, Lili, Comfandi, Primero de Mayo, El Refugio, San Fernando, Santa Isabel, Miraflores, La Cascada, El Lido, Champañangt, Colseguros, entre otros. La comuna 22 es la única comuna que presenta un índice de calidad del empleo alto, la componen los barrios Ciudad Jardín, Parcelaciones de Pance y Urbanización Rio Lili.

Se observa además que, pese a que las zonas de ladera y oriente que son las que presentan valores más bajos de la calidad del empleo, la baja calidad de las condiciones laborales afecta de manera no sistemática a la ciudad y está atomizada por todo el municipio. 
Tabla 4. Resultados del ICE

\begin{tabular}{|c|c|c|}
\hline & Comuna & $\mathrm{ICE}$ \\
\hline \multirow{18}{*}{ Calidad Baja } & Comuna 20 & 0 \\
\hline & Comuna 12 & 16,0753644 \\
\hline & Comuna 13 & 17,3452557 \\
\hline & Comuna 16 & 20,999412 \\
\hline & Comuna 1 & 21,8581118 \\
\hline & Comuna 15 & 22,3092624 \\
\hline & Comuna 14 & 22,4972169 \\
\hline & Comuna 3 & 23,9997123 \\
\hline & Comuna 4 & 26,5655893 \\
\hline & Comuna 11 & 28,208763 \\
\hline & Comuna 21 & 30,1142456 \\
\hline & Comuna 7 & 40,9251167 \\
\hline & Comuna 18 & 42,1019387 \\
\hline & Comuna 9 & 46,0740949 \\
\hline & Comuna 6 & 46,8082642 \\
\hline & Comuna 8 & 53,0715711 \\
\hline & Comuna 2 & 53,9317214 \\
\hline & Comuna 10 & 58,2649535 \\
\hline \multirow{3}{*}{ Calidad Media } & Comuna 5 & 62,3389554 \\
\hline & Comuna 19 & 71,1436318 \\
\hline & Comuna 17 & 78,8868596 \\
\hline Calidad Alta & Comuna 22 & 100 \\
\hline
\end{tabular}

Fuente: cálculos propios a partir de la Encuesta de Calidad de Vida y Empleo, Cali, Ministerio de Trabajo.

La tabla 5, muestra que del total de personas en calidad alta, el 51\% pidió ayuda a familiares y /o amigos, y que esta proporción se incrementa en 7 puntos porcentuales para la calidad del empleo media y llega al $63 \%$ en calidad del empleo baja, las personas con menor calidad del empleo hacen mayor uso de esta canal de búsqueda de empleo. 


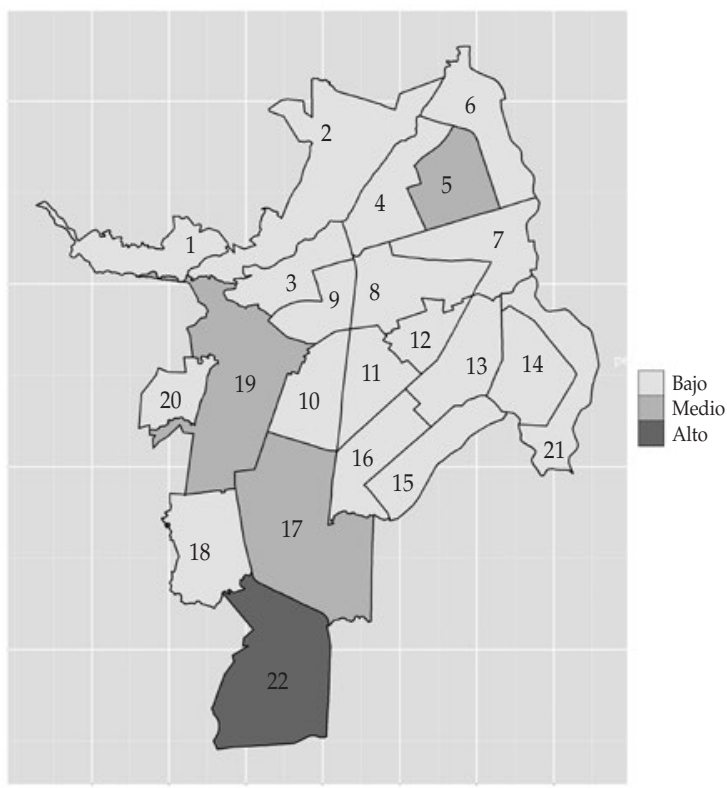

Figura 1. Calidad del empleo en las comunas de Santiago de Cali

Fuente: Cálculos Propios a partir de la Encuesta de Calidad de Vida y Empleo Cali, Ministerio de Trabajo.

Tabla 5. Porcentaje de personas con calidad del empleo alta, media y baja que pidieron ayuda a amigos y/o familiares

\begin{tabular}{cccc}
\hline Pidió(ayuda) Referencias a familiares y/o amigos & Calidad alta & Calidad media & Calidad Baja \\
\hline No & $49 \%$ & $42 \%$ & $37 \%$ \\
Sí & $51 \%$ & $58 \%$ & $63 \%$ \\
\hline
\end{tabular}

Fuente: cálculos propios a partir de la Encuesta de Calidad de Vida y Empleo Cali- Ministerio de Trabajo.

Para analizar los determinantes de la calidad del empleo en las comunas de Santiago de Cali, se procedió a estimar un modelo de variable dependiente multinomial descrito por las ecuaciones (7) y (8) para 10487 individuos ocupados en la ciudad de Cali. Para ello se congregaron las comunas en diferentes grupos: conglomerado Oriente, centro Oriente, centro Norte, ladera y corredor Norte Sur, dicha clasificación es tomada del segundo informe de discriminación laboral en Cali en 2012, el cual toma la clasificación del CIDSE (2011), ${ }^{2}$ donde se establece

2 El CIDSE (2011) establece que las categorías de conglomerados urbanos hacen referencia a una agregación de comunas que ordenan el territorio municipal bajo los siguientes criterios: a) una contigüidad espacial con características geográficas similares (por ejemplo, área de 
la siguiente división geográfica de Cali: conglomerado Oriente, comunas 7, 13, 14, 15 y 21; centro Oriente, 8, 11, 12 y 16; centro Norte, 3, 4, 5, 6, 9 y 10; laderas, comunas 1,18 y 20; finalmente en el corredor Norte y Sur se encuentran las comunas 2, 17, 19 y 22.

A continuación, se estimó el probit ordenado cuya variable dependiente consiste en la calidad del empleo, que es una variable discreta ordenada y como variables independientes la variable afro toma el valor de 1, si el individuo se considera afrodescendiente y cero en otro caso; el sexo toma el valor de 1 si se es hombre y de cero si se es mujer; el estado civil toma el valor de 1 si el individuo se encuentra casado o vive en unión libre; la variable jefe de hogar toma el valor de 1 si el individuo es jefe de hogar y cero en caso contrario. Las variables asociadas a la educación son binarias, que corresponden al máximo título alcanzado en la educación media, técnica, y superior. Se incluyen también dummies por sectores del código CIIU e interacciones entre el canal informal de búsqueda de referencias laborales y las comunas. Sin embargo, es necesario que este modelo cumpla el supuesto de regresiones paralelas.

Tabla 6. Efectos marginales probit ordenado generalizado

\begin{tabular}{lrrr}
\hline & Variable & \multicolumn{3}{c}{ Efectos Marginales } \\
\cline { 2 - 4 } & Calidad alta & Calidad media & Calidad baja \\
\hline Edad & $0,0003086^{* * * *}$ & $0,0007107^{* * *}$ & $-0,0010192^{* * *}$ \\
Oriente & $-0,1300446^{* * *}$ & $-0,1124352^{* * *}$ & $0,2424798^{* * *}$ \\
Centro Norte & $0,3945765^{* * *}$ & $-0,4307587^{* * *}$ & $0,0361821^{* * *}$ \\
Centro Oriente & $0,6117471^{* * *}$ & $-0,3411259^{* * *}$ & $-0,2706212^{* * *}$ \\
Norte Sur & $0,9444918^{* * *}$ & $-0,4682554^{* * *}$ & $-0,4762364^{* * *}$ \\
Sexo & $-0,0036313$ & $-0,0161047^{* * *}$ & $0,019736^{* * *}$ \\
Jefe de hogar & $-0,0103356^{* * *}$ & $-0,0047934^{* * *}$ & $0,0151291^{* * *}$ \\
Estado civil & $0,0081825^{* * *}$ & $-0,0235654^{* * *}$ & $0,0153829^{* * *}$ \\
Afro & $-0,0184777^{* * *}$ & $-0,0098437^{* * *}$ & $0,0283214^{* * *}$
\end{tabular}

ladera) y distribución espacial a partir del centro urbano (centro, centro oriente, oriente); b) distribución urbano-rural de la población; c) el peso demográfico de la población afrodescendiente por comunas y sector censal de acuerdo con los datos del Censo 2005, por considerar este peso demográfico un importante indicador de segregación espacial socio-racial del territorio municipal asociado con la geografía urbana. Dado que el objetivo de este estudio es determinar cuáles son las condiciones en las que se encuentra la calidad del empleo para la población afro y cuáles son los factores que la determinan, se optó por seguir esta distribución la cual incorporaba una ponderación de la importancia de la población afro en cada comuna. 


\begin{tabular}{|c|c|c|c|}
\hline \multirow{2}{*}{ Variable } & \multicolumn{3}{|c|}{ Efectos Marginales } \\
\hline & Calidad alta & Calidad media & Calidad baja \\
\hline Técnico/tecnólogo & $0,0410947^{* * *}$ & $-0,0139809^{* *}$ & $-0,0271139 * * *$ \\
\hline Universitario & $0,0574945^{* * *}$ & $0,010235^{* * *}$ & $-0,0677295^{* * *}$ \\
\hline Posgrado & $0,0561798^{* * *}$ & $-0,0234064^{* * *}$ & $-0,0327734^{* *}$ \\
\hline Pidió referencias a familiares y/o amigos & $-0,0168834^{* * *}$ & $-0,007211^{* * *}$ & $0,0240944^{* * *}$ \\
\hline Referencias*Norte Sur & 0,0051443 & 0,0021347 & $-0,007279$ \\
\hline Referencias*Oriente & $-0,028932^{* * *}$ & $-0,0174792^{* * *}$ & $0,0464111^{* * *}$ \\
\hline Referencias ${ }^{*}$ centro Oriente & $0,0425278^{* * *}$ & $0,0093785^{* * *}$ & $-0,0519063^{* * *}$ \\
\hline Referencias centro Norte & $-0,0238759^{* * *}$ & $-0,0770324^{* * *}$ & $0,1009083^{* * *}$ \\
\hline Industria manufacturera & $0,0090214^{* *}$ & $0,0035939^{* *}$ & $-0,0126153^{*}$ \\
\hline Construcción & $-0586574^{* * *}$ & $0,0319239^{* * *}$ & $0,0267336^{* * *}$ \\
\hline Comercio & $0,0097702^{*}$ & $-0,0268426^{* * *}$ & $0,0170723^{* *}$ \\
\hline Hoteles y restaurantes & $-0,0064871$ & $-0,0032281$ & 0,0097152 \\
\hline Transporte/almacenamiento & $0,0286856^{* * *}$ & $-0,0264071^{* * *}$ & -0022785 \\
\hline Intermediación financiera & $-0,0029318$ & $-0,0013782$ & 0,00431 \\
\hline Actividades inmobiliarias/empresarial & $0,0212321^{* * *}$ & $0,0067445^{* * *}$ & $-0279766^{* * *}$ \\
\hline Administración Pública y Defensa & $0,0437994^{* * *}$ & $0,0078633^{* * *}$ & $-0516628^{* * *}$ \\
\hline Educación & $0,0210591^{* * *}$ & $0,0066245^{* * *}$ & $-0276837^{* * *}$ \\
\hline Servicios Sociales y de Salud & $0,0478278^{* * *}$ & $-0,0312913^{* * *}$ & $-0,0165366^{*}$ \\
\hline $\begin{array}{l}\text { Otras Actividades de Servicios Comuni- } \\
\text { tarios }\end{array}$ & $-0,0005407$ & $-0,0300863^{* * *}$ & $0306269^{* * *}$ \\
\hline Contraste de regresiones paralelas ${ }^{3}$ & 1216,69 & & \\
\hline$\left.{ }^{*}\right)$ significativo al 10\% & & & \\
\hline$(* *)$ significativo al 5\% & & & \\
\hline$(* * *)$ significativo al $1 \%$ & & & \\
\hline
\end{tabular}

Fuente: cálculos propios a partir de la Encuesta de Calidad de Vida y Empleo, Cali, Ministerio de Trabajo.

La tabla 6 muestra los efectos marginales derivados de la estimación de un probit ordenado generalizado. Con excepción de la interacción del con-

3 Dado el estadístico de prueba y el p-valor asociado se rechaza la hipótesis nula en el contraste de regresiones paralelas no se cumple. Dado lo anterior, es necesario estimar un modelo probit multinomial o un modelo probit ordenado generalizado (González y Mora, 2013). 
glomerado de comunas Norte Sur, con la variable binaria de pedir referencias laborales a familiares y /o amigos y las clasificaciones por actividad económica para intermediación financiera, hoteles y restaurantes, todas las variables son estadísticamente significativas. Se observa que un año más de edad aumenta la probabilidad de tener un empleo de calidad alta y media en $0,03 \%$ y $0,07 \%$ respectivamente y disminuye la probabilidad de tener un empleo de calidad baja en $0,10 \%$.

Por otro lado, el hecho de vivir en el Oriente de la ciudad reduce las probabilidades de tener un empleo de calidad media o alta en $13 \%$ y $11 \%$ e incrementa la posibilidad de adquirir un empleo en malas condiciones en aproximadamente un $24 \%$. En relación con las personas que viven en el centro Norte, centro Oriente o en el corredor Norte Sur, respecto a las personas que viven en una zona de ladera, la posibilidad de tener empleos de media y baja calidad disminuye, mientras que la de adquirir empleos de calidad superior se acrecienta. Respecto al sexo, disminuye la probabilidad de tener empleos de media y alta calidad e incrementa la probabilidad de tener una mala calidad del empleo. Con relación con los jefes de hogar, la probabilidad de adquirir empleos de mala calidad se incrementa en 1,5\% mientras que la probabilidad de tener un trabajo de media o alta calidad se reduce en $0,4 \%$ y $1 \%$ respectivamente. El estado civil, casado o en unión libre, reduce la probabilidad de tener un empleo de calidad media $3,3 \%$ y acrecienta la de tener un empleo de altas y malas condiciones en $0,8 \%$ y 1,5\%, respectivamente. Con respecto a la educación, tanto el haber adquirido un diploma de educación media o técnica como universitaria incrementa la probabilidad de tener empleos de calidad alta o media y reduce la de tener un empleo de mala calidad.

Como se puede observar en la tabla 6 , ser afrodescendiente reduce las probabilidades de tener empleos de media y alta calidad en alrededor del 1,8\% y $1 \%$ e incrementa la posibilidad de tener un empleo de mala calidad en $2,8 \%$.

Pedir ayuda a familiares y/o amigos reduce la posibilidad de tener condiciones altas y medias de calidad del empleo en $1,6 \%$ y $0,7 \%$ y acrecienta la de tener malas condiciones en $2,4 \%$. Si se reside en el Oriente o en centro Norte y además, se ha obtenido el empleo mediante la ayuda de amigos y / o familiares, se reduce la probabilidad de tener empleos de alta y media calidad y se incrementa la de tener malas condiciones laborales, situación contraria a los ocupados que residen en el conglomerado centro Oriente.

En relación con la actividad económica, los ocupados que se desempeñan en los sectores construcción, hoteles y restaurantes y servicios comunitarios tienen menos probabilidades de tener empleos de alta calidad y son más propensos a adquirir empleos de mala calidad. A excepción de la actividad de intermediación financiera, la cual no tiene un efecto significativo, los ocupados con empleos en 
las actividades económicas restantes tienen mayor probabilidad de encontrarse con unas condiciones altas y medias de calidad del empleo.

\section{Conclusiones}

La calidad del empleo es un tema de interés, tanto para los economistas como para los tomadores de decisiones, ya que afecta el bienestar y la calidad de vida de los individuos. El análisis de la calidad del empleo es importante, en tanto el gobierno no debe promover solo la generación de empleo sino la generación de empleos de calidad (Weller et al., 2012; González \& Mora, 2011).

Los resultados del cálculo del ICE para las 22 comunas de Santiago de Cali muestran que la comuna que mejor índice de calidad del empleo presenta es la comuna 22, con una valor de 100. Posteriormente se encuentran las comunas 5, 19 y 17, conformando las comunas con una calidad del empleo media. Finalmente, 18 de las 22 comunas presentaron un valor bajo de la calidad del empleo estas son las comunas 20, 12, 13, 16, 1, 15, 14, 3, 4, 11, 21, 7, 18, 9, 6, 8, 2 y 10 . Esto muestra que, pese a que el problema de la calidad del empleo se intensifica en algunos sectores (ladera y Oriente), este se encuentra atomizado por todo el municipio.

Uno de los factores que inciden en la calidad del empleo es el lugar de residencia, encontrándose que el hecho de residir en una de las comunas que pertenecen al Oriente hace que la probabilidad de tener malas condiciones laborales se incremente en el $24 \%$, mientras que las de tener un empleo de media o alta calidad se reduce en $11,2 \%$ y $13 \%$, respectivamente.

Respecto a los sectores de actividad económica, si bien no se puede asegurar que existen clústeres de calidad del empleo, los ocupados que se desempeñan en los sectores construcción, hoteles y restaurantes y servicios comunitarios tienen menos probabilidades de tener empleos de alta calidad y son más propensos a adquirir empleos de mala calidad.

Por otro lado, pedir ayuda (referencias laborales) a familiares y/o amigos reduce la probabilidad de tener empleos de alta y media calidad e incrementa la probabilidad de encontrarse en malas condiciones laborales, en especial si se reside en sectores específicos de la ciudad, como el conglomerado Oriente, caracterizado por ser una zona deprimida de la ciudad. Por tanto, siguiendo a Bayer, Ross y Topa (2005) y Brock y Durlauf (2002), es posible que individuos que viven en zonas deprimidas de la ciudad conformen redes sociales deterioradas que les transmitan información acerca de empleos de mala calidad, formando un efecto negativo dada la mala calidad de la información transmitida, 
el $63 \%$ de las personas con condiciones precarias del empleo acudió al uso de referencias laborales.

Para los individuos afrodescendientes se reducen las probabilidades de tener empleos de media y alta calidad en alrededor del 1,5\% e incrementa la posibilidad de tener un empleo de mala calidad en 2,8\%, situación que refuerza la discriminación por raza que existe en el mercado laboral de la ciudad de Santiago de Cali. En Cali la condición étnica afrodescendiente limita el acceso a empleos de alta calidad, estos resultados son acordes a lo encontrado por Bustamante y Arroyo (2008) en Santiago de Cali. Esta situación se ve agravada por los bajos niveles educativos de la población en ciertos sectores de la ciudad y por la existencia de localizaciones geográficas de la calidad del empleo.

Al igual que Mora y González (2011) y Guataqui, Camacho y Dussán (2012), los resultados muestran que la educación incrementa la probabilidad de tener empleos de calidad, por tanto, el fortalecimiento de la educación y la capacitación de los recursos humanos puede constituir una estrategia para la creación activa de empleo digno en la ciudad de Cali. En este orden de ideas, los resultados de las estimaciones realizadas en este artículo muestran que la educación, tanto para la población total como para la población afro y la no afro, tiene un efecto positivo y significativo como factor determinante de la calidad del empleo. Programas de capacitación y de ingreso a la educación superior generarían cambios positivos en la calidad del empleo de los individuos en general y en especial en los de tez negra. Además, siguiendo a Guataqui, Camacho y Dussán (2012), políticas de retención de la población en edad escolar y la evaluación de la calidad y pertinencia de la educación superior y la formación para el trabajo acompañada con políticas dirigidas al incremento de la productividad empresarial que absorban la mano de obra, podrían generar que individuos más capacitados encuentren empleo de mejor calidad en un mercado laboral más dinámico.

\section{Referencias}

Arroyo, S., \& Bustamante, C. (2008). La raza como determinante del acceso a un empleo de calidad: un estudio para Cali. Ensayos sobre Política Económica, 26(57), 130-175.

Bescond, D., Chataignier, A., \& Mehran, F. (2003). Seven indicators to measure decent work: An international comparison. International Labor Review, 142(2), 179-212.

Bonnet, F., Figuereido, J., \& Standing, G. (2003). A family of decent work indexes. International Labor Review, 142(2), 213-238. 
Brant, R. (1990). Assessing Proportionality in the Proportional Odds Model for Ordinal Logistic regression. Biometrics, 46(4), 1171-1178.

Cameron, A. C., \& Trivedi, P. K. (2005). Microeconometrics. Cambridge: Cambridge University Press.

CIDSE. (2011). ¿Cuántos Sómos? ¿Cómo Vamos? Cali: Diagnóstico Sociodemográfico de Cali y 10 municipios del Pacífico nariñense. Afroamérica XXI. Recuperado de http:/ / www.dane.gov.co/files/censo2005/etnia/sys / cuantos_somos.pdf

Farné, S. (2003). Estudio sobre la calidad del empleo en Colombia. Estudios sobre economía laboral en Colombia, 5.

Farné, S. (2012). La calidad del empleo en América Latina a principios del siglo XXI, una mirada especial a los casos de Bolivia, Chile, Colombia, Ecuador y Perú. En Farné, S. (Comp.), La Calidad del Empleo en América Latina a principios del siglo XXI (pp. 15-32). Bogotá: Universidad Externado de Colombia.

Ghai, D. (2003). “Trabajo Decente Conceptos e Indicadores”. Revista Internacional del Trabajo, 122(2), 125-160.

Greene, W. H., \& Hensher, D. A. (2010). Modeling Ordered Choices. Cambridge: Cambridge University Press.

González, S., \& Guillén, A. M. (2009). La calidad del empleo en la Unión Europea. Debate político y construcción de indicadores. Revista del ministerio de Trabajo e Inmigración, 81, 71-88.

Guataqui, J. C., Camacho, C. X., \& Dussán, L. F. (2012). Calidad del Empleo en Bogotá una Aproximación Desde el Enfoque de Trabajo Decente. Cuadernos de Desarrollo Económico, 16.

Lanari, M. E. (2005). Trabajo Decente: Significados y alcances del concepto. Indicadores propuestos para su medición. En: Trabajo, ocupación y empleo. Relaciones laborales y grupos particulares de actividad. Buenos Aires: Subsecretaria de Programación Técnica y Estudios Laborales.

Levaggi, V. (2006). Democracia y Trabajo Decente en América Latina. Lima: Organización Internacional del Trabajo.

Long, J. S. (1997). Regression Models for Categorical and Limited Dependent Variables. Thousand Oaks, CA: SAGE Publications, Inc.

Mora, J. J. \& González, C. G. (2013). Aprendiendo microeconometría con Sata. Working-paper. Cali: Universidad Icesi.

Mora, J. J., \& Ulloa, M. P. (2011). Calidad del empleo en las principales ciudades colombianas y endogeneidad de la educación. Revista de economía institucional, 13(25), 163-177.

Mora, J. J., \& González, C. G. (2011). Políticas Activas de Empleo para CaliColombia. Estudios Gerenciales, 27(128), 13-41. 
OIT (1999). Trabajo Decente. Memoria del director general a la 87 reunión de la conferencia internacional del trabajo. Ginebra. Recuperado de http:/ / www.ilo. org/public/spanish/standards/relm/ilc/ilc87/rep-i.htm

Peters, W., \& Butler, J. (1970). The construction of Regional Economic Indicators by principal components. Annals of Regional Science, IV, 1-14.

Pineda, J. (2011). Calidad del trabajo: aproximaciones teóricas y estimación de un índice compuesto, Ensayos sobre política económica, 65, 60-105.

Posso, Ch. M. (2012). Calidad del empleo y segmentación laboral: un análisis para el mercado laboral colombiano 2001-2006, Desarrollo y Sociedad 65, 191-234.

Reinecke, G., \& Valenzuela, E. (2000). La calidad del empleo: un enfoque de género. En M. E Valenzuela \& G. Reinecke (Eds.), ¿Más y mejores empleos para las mujeres? La Experiencia de los países del Mercosur y Chile (pp. 59-101). Chile: OIT

Senbruch, K. (2007). From the Quantity to the Quality of Employment. En S. Alkire, F. Comim \& M. Qizilbash (Eds.), The Capability Approach in Human Development: Concepts Applications and Measurement (pp. 1-57). Ciudad: Berkeley: Cambridge University Press.

Somarriba N., Merino M., Ramos G., \& Negro A. (2010). La calidad del empleo en la Unión Europea. Estudios de economía aplicada, 28(3), 1-22.

Somavia, K. (2004). A fair globalization. The role of the ILO. Report of the DirectorGeneral on the Commission on the social dimension of globalization. International Labour Organization- Geneva.

Tenjo, J. \& Herrera, P. (2009). Dos ensayos sobre discriminación: discriminación salarial y discriminación en acceso al empleo por origen étnico y por género. Bogotá: Universidad Javeriana. Recuperado de http:/ / www.javeriana.edu.co/ fcea/pdfs_depto_economia/paper_2009_02_dos_ensayos_sobre_discriminacion.pdf

TMDMDW (septiembre, 2008). Medición del trabajo decente. Documento de debate para la Reunión tripartita de expertos sobre la medición del trabajo decente. Ginebra.

Uriarte, E. (2001). Trabajo decente y formación profesional. Boletín Cinterfor, 151, 9-26.

Viáfara, C. A., \& Urrea, F. (2006). Efectos de la raza y el género en el logro educativo y estatus socio-ocupacional para tres ciudades colombianas. Desarrollo y Sociedad, 115-163.

Weller, J. \& Roethlisberger, C. (2012). La Calidad del empleo en América Latina: Un análisis conjunto. En Farné, S. (Comp.), La Calidad del Empleo en América Latina a principios del siglo XXI (pp. 33-118) Bogotá: Universidad Externado de Colombia. 


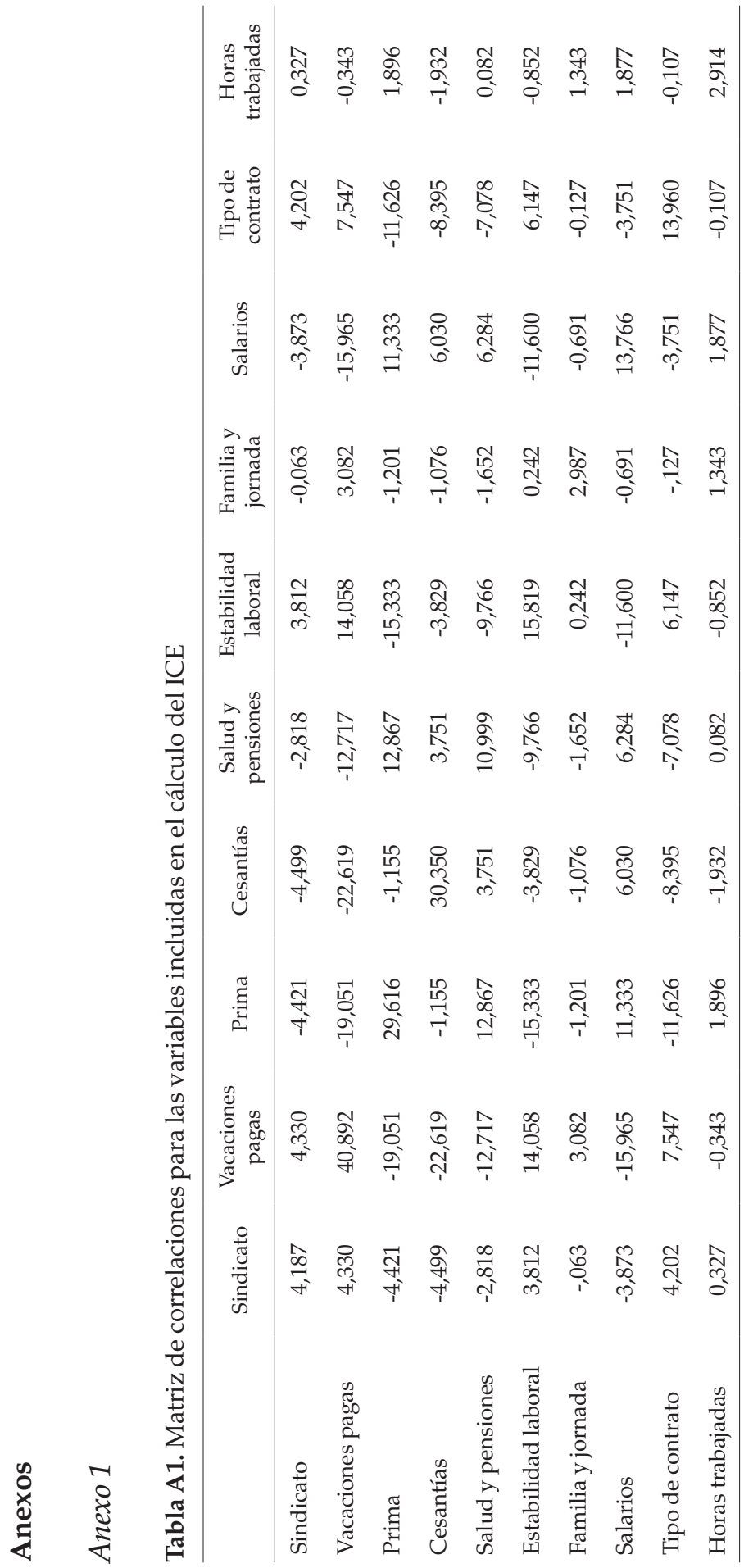




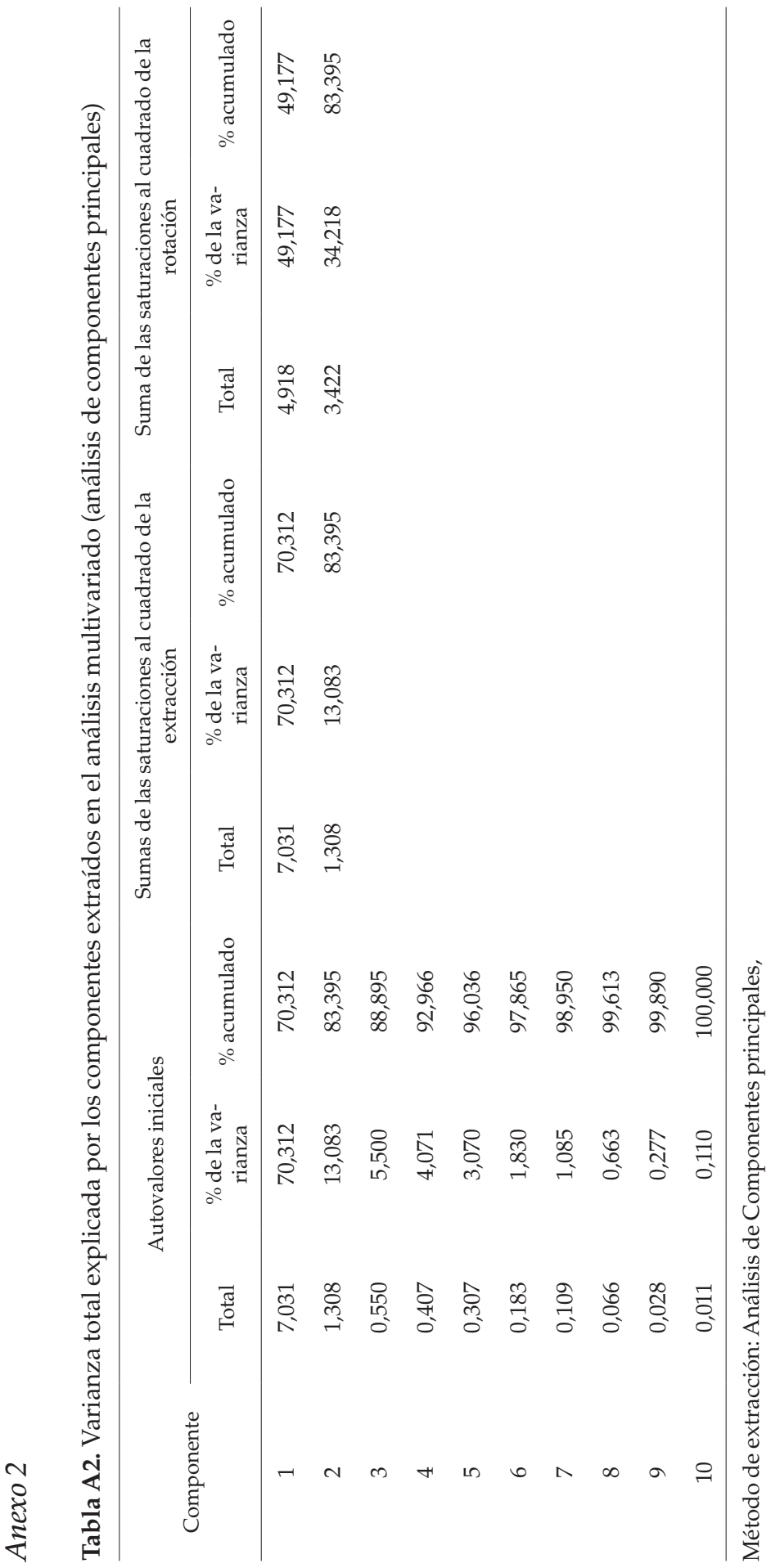




\section{Anexo 3}

Tabla A3. Matriz de componentes rotados (Varimax)

\begin{tabular}{lcc}
\hline & \multicolumn{2}{c}{ Componente } \\
\cline { 2 - 3 } & 1 & 2 \\
\hline Sindicato & 0,502 & 0,607 \\
Vacaciones pagas & 0,919 & 0,331 \\
Prima & 0,906 & 0,309 \\
Cesantías & 0,950 & 0,241 \\
Salud y pensiones & 0,708 & 0,514 \\
Estabilidad laboral & 0,628 & 0,653 \\
Familia y jornada & 0,258 & 0,824 \\
Salarios & 0,525 & 0,748 \\
Tipo de contrato & 0,924 & 0,203 \\
Horas trabajadas & & $-0,905$ \\
\hline
\end{tabular}

Método de extracción: Análisis de componentes principales.

Método de rotación: Normalización Varimax con Kaiser. 
